\title{
The deforming effects of stellar magnetic fields on spectral line profiles
}

\author{
Ewald Gerth ${ }^{1}$ and Yurij V. Glagolevskij ${ }^{2}$ \\ ${ }^{1}$ D-14471 Potsdam, Gontardstr. 130, Germany \\ email: ewald-gerth@t-online.de \\ ${ }^{2}$ Special Astrophysical Observatory, Nizhnij Arkhyz, Karachaevo-Cherkesia, 369167 Russia. \\ email: glagol@sao.ru
}

\begin{abstract}
The profile of a stellar spectral line is formed by the transfer of radiation through the atmosphere by atomic processes in different chemical elements distributed usually unequally over the surface of a magnetic star.

The theory of model atmospheres accounts for all possible physical conditions. Usually one assumes chemical homogeneity with a plane parallel atmosphere. The resulting line profile, however, is strongly deformed by the geometrical influence of the topographic element distribution and the magnetic surface field structure as well as the projection onto the line of sight of the outgoing radiation from all surface points and its integration over the visible disk.

Line formation by the geometry of projection and element distribution is used for the inverse procedure of Doppler Imaging by V .L. Khokhlova and her followers. We consider here only the influence of the magnetic field on the line profile including the Stokes parameters $I, Q, U, V$, which we study separately from other effects. Thus as a function of the stellar aspect due to rotation the magnetic field and the projection make a symmetric "plane atmosphere profile" asymmetric.

Line profile deformation by the magnetic field leads to a fatal consequence for the traditional measurement of stellar magnetic fields by the Zeeman displacement of the circularly polarized $\sigma$-components. The large scatter of measuring points is partly due to the asymmetry of the line profiles!
\end{abstract}

Keywords. Line: formation, line: profiles, stars: magnetic fields, techniques: polarimetric

\section{The formation of spectral line profiles}

Observation of the integral magnetic field of a star's surface is given as phase curves and line profiles of polarized light in the four Stokes parameters $I, Q, U, V$. Our algorithms are described by Gerth et al. (2001) and more completely by Gerth \& Glagolevskij (2004).

The observed line profile is formed by several distinct physical processes:

1. Radiative transfer with atomic interaction in the stellar atmosphere

2. Microturbulence (thermic motion)

3. Macroturbulence (convection)

4. Flows (circular, meridional, and equatorial currents)

5. Observational and instrumental conditions (seeing, optics of the telescope and of the spectrograph, resolution of storage, reduction, etc.)

6. Rotation (including inclined and differential rotation)

7. Geometry of the radiating parts of the stellar surface seen by the observer in integrated light

These processes together contribute to a vast mixing of influences. As far as convolution is involved, we can analyze single broadening profiles, like the so-called instrumental profile. We combine all profiles, which broaden the radiative transfer profile into a profile 
function $\omega$, which comprises processes 1-5. Process 6 is connected with the radial velocity, which shifts the line profile in wavelength and has to be treated with the Zeeman displacement due to the magnetic field.

In this paper we emphasize the influence of the geometry (process 7). Hitherto, line profile formation has been investigated mainly with plane atmosphere layers, which do not account for the spherical stellar geometry. We restrict consideration of line profile formation to only geometrical conditions, which we will analyze separately from all other influences.

\section{The effect of integration of the radiation flux}

Usually we measure the (integrated) magnetic field from the Zeeman displacement of the line profiles of oppositely circularly polarized light. What we call the effective magnetic field $B_{\text {eff }}$ is not a mean value but is the result of weighting and of convolving the radiation flux containing magnetic field information concerning the form and the position of the profiles of all surface elements in which the line formation process takes place.

For the visibility of the star by the observer, we define a window function $w(i, \varepsilon, \delta, \varphi)$, containing the inclination $i$, the projection of each surface element onto the line of sight, and the limb darkening with its angular distance $\varepsilon$ from the center of the visible disk, which averages and normalizes the vector $\boldsymbol{B}(r, \delta, \varphi)$ with the orthogonal components $B_{r}$, $B_{f}, B_{d}$ of the magnetic surface field:

$$
\boldsymbol{B}_{\text {int }}(t)=\frac{\int_{\delta=-\pi / 2}^{\pi / 2} \int_{\varphi=0}^{2 \pi} \boldsymbol{B}(r, \delta, \varphi) w(i, \varepsilon, \delta, \varphi-t) \mathrm{d} \varphi \mathrm{d} \delta}{\int_{\delta=-\pi / 2}^{\pi / 2} \int_{\varphi=0}^{2 \pi} w(i, \varepsilon, \delta, \varphi-t) \mathrm{d} \varphi \mathrm{d} \delta}
$$

This integral formula gives the integrated mean of the disk seen by the observer and comprises the convolution integral, which represents the rotation of the star with its map $B(\varphi, \delta)$ behind the window $w(i, \varepsilon, \delta, \varphi) . t$ is set equal to the longitude $\varphi$ and characterizes the rotation of the momentary orientation angle at the longitude $\varphi$ as a function of time. The denominator makes the normalization.

The line profile is found by a further integration. The distribution of the polarized radiation over a region $b$ around $\boldsymbol{B}$ is defined by $\omega(b)$ and convoluted with the phase integral equation (2.1):

$$
\boldsymbol{B}_{\text {int }}(t, b)=\frac{\int_{\delta=-\pi / 2}^{\pi / 2} \int_{\varphi=0}^{2 \pi} \int_{-\infty}^{+\infty} \boldsymbol{B}(r, \delta, \varphi, \beta) w(i, \varepsilon, \delta, \varphi-t) \omega(\beta-b) \mathrm{d} \beta \mathrm{d} \varphi \mathrm{d} \delta}{\int_{\delta=-\pi / 2}^{\pi / 2} \int_{\varphi=0}^{2 \pi} \int_{\infty}^{+\infty} w(i, \varepsilon, \delta, \varphi-t) \omega(\beta-b) \mathrm{d} \beta \mathrm{d} \varphi \mathrm{d} \delta}
$$

Equation (2.2) is the analytical representation of the geometric line profile at a given phase, containing the convolutions due to rotation and to the frequency distribution of the field strength. In the computation program the integrals are calculated as matrices.

For the investigation of the geometrical profile without considering other line forming processes, we set the distribution function to a $\delta$-function

$$
\omega(b)=\delta(\beta-b) .
$$



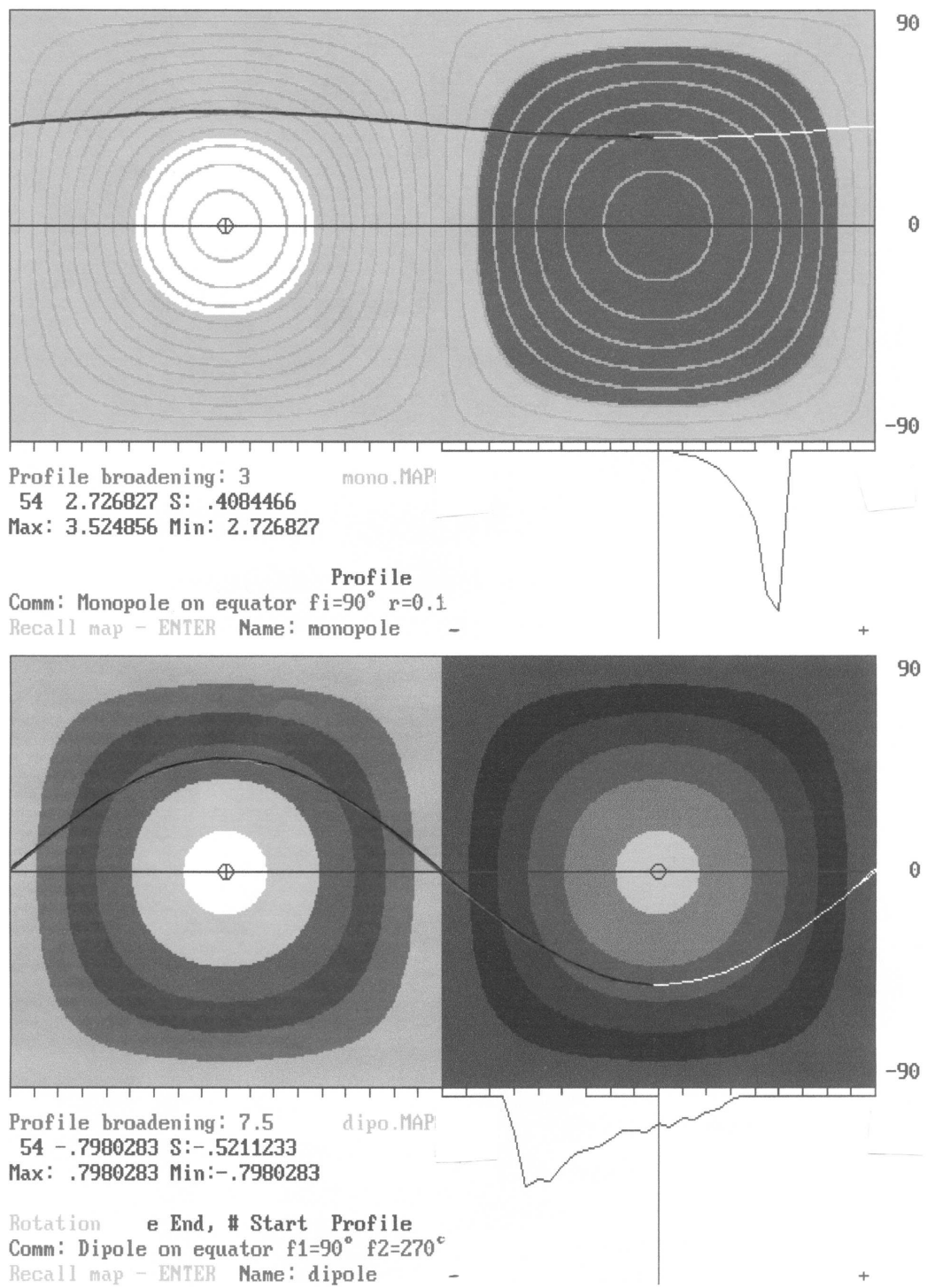

Figure 1. Magnetic maps with coordinated phase curves and line profiles. Demonstration of computer screen: (Colored) maps with iso-magnetic lines and areas; manipulating menu (in this reproduction only indicated); the line profile is moving on the screen with the phase of rotation. The profile is shown at phase 0.75 , marked by the change of the phase curve from black to white. Left panel: Magnetic monopole in one hemisphere.

\section{Parameters:}

$$
\begin{array}{clll}
\text { Radius-fraction } & \text { Longitude } & \text { Latitude } & \text { Charge } \\
r=0.5 & \varphi=90^{\circ} & \delta=+45^{\circ} & Q=+1
\end{array}
$$

The field is always positive with a minimum at $\varphi=270^{\circ} \delta=0^{\circ}$. The line profile is asymmetric and has its deepest point at the field minimum phase 0.75 .

Right panel: Central magnetic dipole with separated magnetic charges. Parameters:

$$
\begin{array}{cccl}
\text { Radius-fraction } & \text { Longitude } & \text { Latitude } & \text { Charge } \\
r_{1}=0.5 & \varphi_{1}=90^{\circ} & \delta_{1}=+45^{\circ} & Q_{1}=+1 \\
r_{2}=0.5 & \varphi_{2}=270^{\circ} & \delta_{2}=-45^{\circ} & Q_{2}=-1
\end{array}
$$

The line profile changes polarity at phases 0.0 and 0.5 with a nearly rectangular form and shows at the poles an extreme asymmetry at phase 0.75 with a steep edge. 


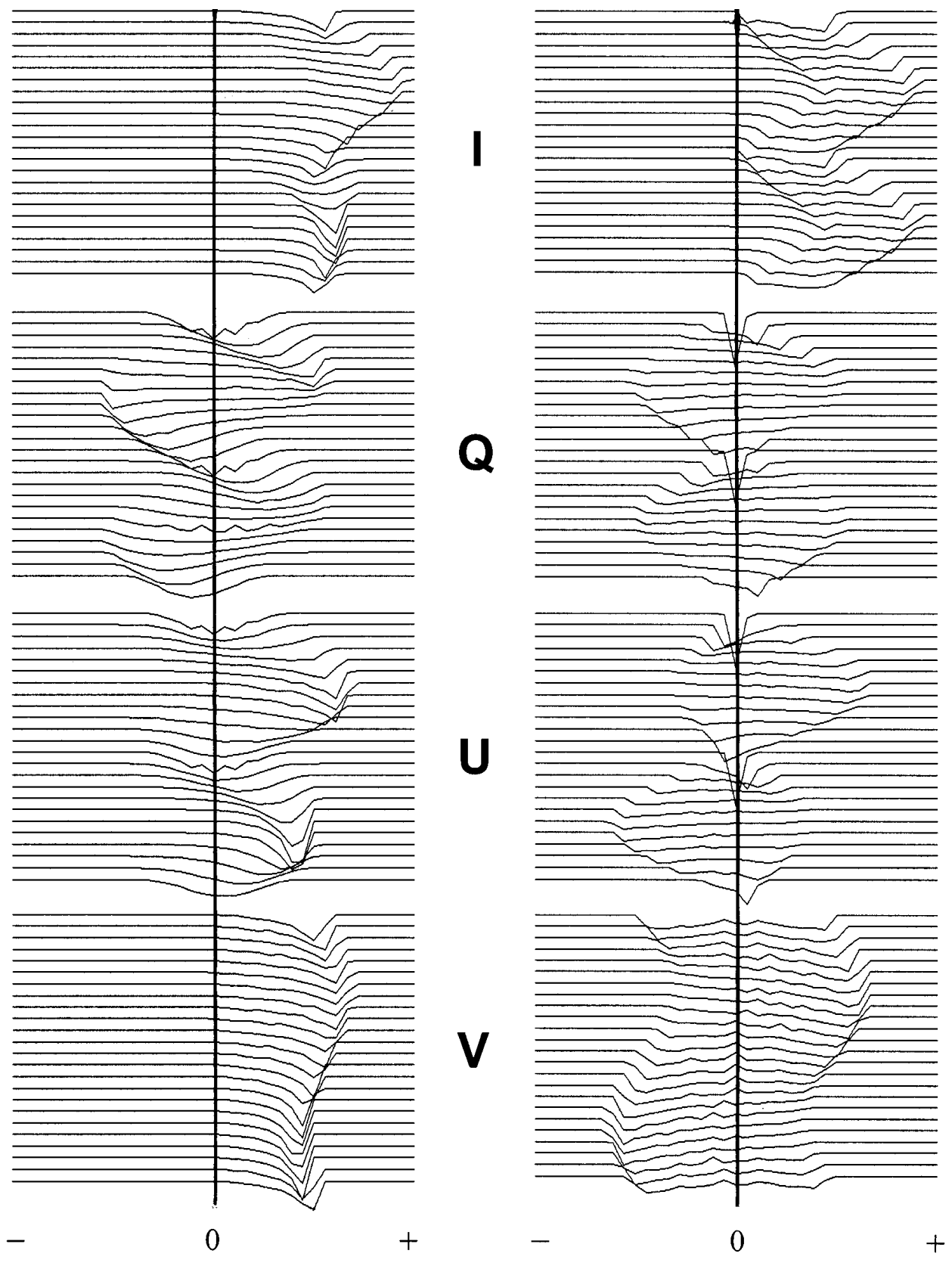

Figure 2. A series of geometrical line profiles of a monopole and a dipole for the Stokes parameters $I, Q, U, V$. Abscissa: Definition range of frequency distribution. Ordinate: phase $0 \cdots 1$, phase step 0.05. The dipole profiles emerge by the linear superposition of two monopole fields of opposite polarity. Left panel: A virtual magnetic monopole is investigated by its Stokes profiles. The parameters $I$ and $V$ reflect best the expected course of the field; $Q$ shows a double wave with a change of polarity; $U$ is only positive; $I, U$ and $V$ have steep edges at their positive side. The line profiles are asymmetric and vary considerably with phase. Right panel: Stokes profiles of a magnetic dipole consisting of two magnetic charges in the equatorial plane. The parameters $I$ and $V$ for a dipole reflect also best the expected course of the field. Besides the bumping-like double wave, at $Q$ and $U$ the variability of the wavelength with a change of polarity and asymmetry is conspicuous. 


\section{The geometrically caused asymmetry of line profiles}

The geometrical line profiles are usually asymmetric and deviate from the normal (Gaussian) distribution. The maximum and the center of gravity do not coincide. Contrary to what we might think, the maximum of the integral magnetic field strength is not when the star seen pole-on, but by the greatest magnitude of the integral over the disk of all vector field components directed toward the line of sight of the observer. If all vectors in the surface elements are nearly parallelly directed, then there would occur a sharp maximum; otherwise, in the case of a wide-spread distribution over the surface the maximum would produce a broad profile.

The profile form depends strongly on the local position on the stellar surface the observer sees. During the star's rotation, the beam viewed by the observer slides along the latitude circle with the inclination angle $i$, producing thus a periodically varying geometrical profile with characteristic deviations from symmetry.

\section{Consequences for the measurement of magnetic fields by the form and the spectral location of line profiles}

The asymmetry of the line profiles has serious consequences for the measurement of magnetic fields by the Zeeman-shift between the $\sigma$-components of the circularly polarized light. Every observer (including the authors with their experience of three decades) is inclined to measure a line by the spectral position of the maximum or by the best visual correlation of the profile with its mirror image. In earlier times we could not explain the strange asymmetries of the line profiles seen on the oscilloscope screen. However, with our new knowledge about the general natural asymmetry of the line profile, we can now interpret the uncertainties of our former measurements.

Indeed, our old measurements were not wrong at all, because they reveal at least a statistical tendency for the magnitude of the magnetic field strength and its variation, but they all suffer from a very high scatter, which is not only due to the graininess of the photographic plate, but is primarily caused by the varying aspect of the star.

\section{References}

Gerth E., Glagolevskij Yu.V.: 2001, in: Magnetic fields across the Hertzsprung-Russell diagram, eds.: Mathys G., Solanki S.K., Wickramsinghe D.T., Santiago, Chile, 248, 333

Gerth E., Glagolevskij Yu.V.: 2004, in: Proc. International Meeting and Workshop 27.8.-2.9.2003, SAO Nizhnij Arkhyz, Russia. (in press) 